\title{
Relevancia de la heterogeneidad socioeconómica: Estudio comparativo entre América Latina y Europa basado en la adaptación del Esquema EGP
}

\author{
Idelfonso Marqués Perales \\ Universidad de Sevilla. Departamento de Sociología \\ imarques@us.es
}

\section{Eduardo Chávez Molina}

Universidad de Buenos Aires. Facultad de Ciencias Sociales.

Instituto de Investigaciones Gino Germani

echavez@retina.ar

\section{Resumen}

Los estudios comparativos internacionales de estratificación social y movilidad de clases se basan en esquemas de clases concebidos para los países industrializados. El esquema EGP, desarrollado por Erikson, Goldthorpe y Portocarero, se ha convertido en un estándar en el campo de la movilidad social. Sin embargo, este esquema puede no reflejar heterogeneidades en las relaciones laborales que se han agravado en los últimos años producto de la flexibilización, precarización y pauperización de los contratos, donde las ocupaciones asalariadas y autónomas están segmentadas en sectores de alta y baja productividad, con condiciones de trabajo y remuneraciones desiguales. La hipótesis que guía nuestro trabajo es la siguiente: el impacto de heterogeneidad en América Latina es tan fuerte que esta dimensión ha de ser considerada en el análisis de clase. Nuestra hipótesis alternativa es que en países del sur de Europa se hallan en una situación intermedia entre América Latina y la Europa tempranamente industrializada.

Proponemos una adaptación del esquema EGP para explicar tal heterogeneidad. Para ello, utilizamos datos de encuestas nacionales de hogares en algunos países latinoamericanos y también de países del sur de Europa, con la intención de caracterizar sus estructuras de clase y analizar la asociación entre la pertenencia a la clase y las condiciones de vida.

* Este artículo ha sido elaborado en el marco del proyecto INCASI, financiado por The European Union's Horizon 2020 Research and Innovation Programme, Marie Skłodowska-Curie (GA No 691004). El proyecto está coordinado por el Dr. Pedro López-Roldán (Universitat Autònoma de Barcelona). El artículo refleja solo el punto de vista de los autores, y la agencia no es responsable del uso que se haga de la información que contiene. 
La introducción de distinciones en el esquema de clases es relevante en contextos de alta heterogeneidad, no en todos los contextos económicos, por lo cual es esperable que, en sociedades de mayor desarrollo relativo o viceversa, las clases sociales no sean las mismas.

Palabras clave: heterogeneidad socioeconómica; esquema de clases; desigualdad; pobreza; América Latina; Europa

Abstract. The importance of socio-economic heterogeneity: A comparative approach grounded in the EGP scheme

International comparative studies of social stratification and class mobility are based on class schemes conceived for industrialized countries. The EGP scheme, developed by Erikson, Goldthorpe and Portocarero, has become a standard in the field of social mobility. However, this scheme may not reflect heterogeneities in labor relations that have worsened in recent years as a result of the flexibilization, precarization and pauperization of contracts, where salaried employment and self-employment are segmented into sectors of high and low productivity, with unequal working conditions and wages. The hypothesis that guides our work is the following: the impact of heterogeneity in Latin America is so strong that this dimension must be considered in class analysis. Our alternative hypothesis is that southern European countries are in an intermediate situation between Latin America and early industrialized Europe. We propose an adaptation of the EGP scheme to explain such heterogeneity. For this purpose, we use data from national household surveys conducted in some Latin American countries as well as southern European countries with a view to characterizing their class structures and analyzing the association between class membership and life conditions. The introduction of distinctions in the class scheme is relevant in contexts of high heterogeneity, not in all economic contexts, so it is expected that in societies with greater relative development or vice versa, the social classes are not the same.

Keywords: socio-economic heterogeneity; class scheme; inequality; poverty; Latin America; Europe

\section{Sumario}

1. Introducción 5. Segunda dimensión: la pobreza relativa

2. Razones para comparar

6. Tercera dimensión: tipo de contrato

3. La heterogeneidad laboral 7. Contraste entre ambos esquemas para en el mercado europeo los países europeos y latinoamericanos

4. La heterogeneidad de las clases 8. Conclusiones sociales en Europa

Referencias bibliográficas

\section{Introducción}

Nuestro artículo se propone analizar en forma comparativa algunos países de América Latina y Europa, con la intención de observar las condiciones de sus potenciales heterogeneidades internas que explican procesos de desigualdad y pobreza. Si bien ambas zonas son muy dispares en términos históricos, todas 
ellas han convergido en la necesidad de analizar los nuevos procesos de heterogeneidad estructural que afectan a sus sociedades.

Los estudios comparativos internacionales de estratificación y movilidad social se basan en esquemas de clases concebidos para los países industrializados. Uno de estos esquemas, el esquema EGP, desarrollado por Erikson, Goldthorpe y Portocarero (1979), se ha convertido en un estándar en el campo de la movilidad social. Sin embargo, este esquema puede no reflejar heterogeneidades en las relaciones laborales que se han agravado en los últimos años producto de la flexibilización, precarización y pauperización de los contratos (formales o tácitos), donde las ocupaciones asalariadas y autónomas están segmentadas en sectores de alta y baja productividad, con condiciones de trabajo y remuneraciones desiguales.

Los objetivos que persigue nuestro trabajo tienen un doble cometido. En primer lugar, trataremos de dar cuenta de un esquema de clase que contemple las divisiones propias de las sociedades de América Latina. En segundo lugar, trasladamos este esquema a Europa con el objetivo de comprobar si las innovaciones introducidas valen también para esta región. Dado que este esquema es una adaptación de una clasificación creada en Europa (pero en un periodo de intensa industrialización), esperamos que sirva para analizar los procesos de desigualdad que atenazan las sociedades de América Latina (validez interna). A la vez, esperamos que el esquema nos sirva para esclarecer el papel que cumplen de algunas de las diferencias que establece en Europa (validez externa).

Una de las hipótesis que guía este trabajo es que en el sur de Europa la heterogeneidad importa en mayor medida que en el norte europeo, pero influye menos, porque hay menos brechas de productividad, con relación a lo que sucede en América Latina, que presenta a su vez fuertes heterogeneidades subregionales.

Proponemos una adaptación del esquema EGP para explicar tal heterogeneidad. Para ello, utilizamos datos de encuestas nacionales de hogares de algunos países latinoamericanos, como Argentina, Ecuador, Guatemala y México, y también de Suecia, Reino Unido, Francia y España, en Europa, con la intención de caracterizar sus estructuras de clase y analizar la asociación entre la pertenencia a la clase y las condiciones de vida. La introducción de distinciones en el esquema de clases es relevante en contextos de alta heterogeneidad, no en todos los contextos económicos, por lo cual es esperable que, en sociedades de mayor desarrollo relativo o viceversa, las clases sociales no sean las mismas.

\section{Razones para comparar}

¿Qué utilidad tiene observar a otros para conocernos mejor? Tal vez sea esta la pregunta inicial que permite recorrer a lo largo de este capítulo una mirada comparativa sobre la caracterización y el acontecer de las clases ocupacionales de ciertos países de América Latina y Europa, y sus procesos distributivos de los ingresos captados en encuestas. 
La primera preocupación implica específicamente qué es lo comparable y para qué, qué sentido tiene la comparación y si la misma responde a los objetivos y preocupaciones iniciales del artículo. En ese marco, proveer bajo la mirada analítica comparativa una estructura de medición, que permita observar cuán parecidos o cuán disímiles son las estructuras de clases de los países seleccionados, que identifique, por un lado, las condiciones de la heterogeneidad social y, por otro, si esos rasgos pueden observarse a la luz de las constricciones y austeridad económica europea en la última década.

En ese sentido es necesario acordar para el lector qué es lo comparable, bajo un principio general que atiende al objetivo del artículo de observar las condiciones de sus potenciales heterogeneidades internas que explican procesos de desigualdad y pobreza en ambas regiones, y si esa desigualdad es pertinente, y nos presenta el primer horizonte: una mira interpaíses, pero que a su vez debe reflejar las observaciones intraregiones, para observar el peso que implica la heterogeneidad económica productiva al interior de cada aglomerado.

Ahora bien, también es necesario calibrar los registros de la comparación, que implican, por un lado, los aspectos teoréticos, instrumentales y de resultados preliminares.

Con respecto a la comparación teórica entre América Latina y Europa, implica el uso de conceptos que remitan a un conjunto posible de categorías armoniosamente comprensibles de la misma forma en ambas regiones y países, que posibiliten un diálogo compatible, en un ejercicio temporal determinado. Los conceptos de regiones, países, desigualdad social, distribución, ingresos, heterogeneidad estructural remiten a un conjunto de conceptos comprensibles en áreas territorial e históricamente distintas, pero implican referencias teóricas que han surgido en contextos distintos, basado en preocupaciones societales y científicas específicas, en términos de comprensión y explicación de fenómenos sociales que son llevados al análisis.

Por otro lado, el instrumental metodológico conlleva a armonizar bases de datos, cuestionarios, preguntas específicas y, en base a dichas construcciones, la elaboración de variables que remitan a un proceso homogéneo de medición. Las advertencias quedan en el plano de cualquier fuente secundaria, aunque en este caso sacando provecho de disímiles fuentes de datos, que en el caso latinoamericano no fueron realizados con el afán de la comparación interpaíses, y a diferencia de la Unión Europea, que cuenta con herramientas específicas a nivel continental. ¿¿Cuánto de ello es valedero?, queda al esfuerzo conjunto del proceso de armonización que conlleva a entender y consensuar la generación de variables que pueden ser comparadas, a pesar de las potenciales diferencias de la recolección de datos.

Y por último, la comparación de resultados, que obliga a observar los objetivos del presente artículo, a la necesidad de la vigilancia de las conclusiones, que constituyen parámetros que no solo se incorporan a la propia racionalidad académica, sino a la importancia societal, de mirar con mayor detenimiento los elementos comparativos, y las distancias y convergencias de los procesos de desigualdad y heterogeneidad puestos en observación comparativa por nosotros. 
El recorrido de la comparación gira en torno a los siguientes parámetros:

1. Los recursos económicos por ingreso neto mensual valorizados en una medida homogénea como los dólares PPA (Poder de Paridad Adquisitiva del dólar), según cada clase ocupacional.

2. La pobreza relativa, basada en el criterio de una variable monetaria como es el ingreso. Se ha fijado un nivel mínimo de la variable por debajo del cual las personas serán clasificadas como pobres, y por encima, como no pobres. La variable elegida es el ingreso, el nivel dependerá de la distribución de los ingresos en la población, $60 \%$ por debajo de la mediana de ingresos.

3. El tipo de contrato laboral, a fin de observar la formalización de los asalariados, lo cual remite a mecanismos de seguridad laboral, y temporalidad en la forma de contratación.

Queda en agenda de investigación próxima, las diferencias por género, por subterritorios, las prestaciones extrasalariales referidos los beneficios por fuera del salario de la actividad laboral, entiéndase la misma como seguro médico, vacaciones, pagas adicionales, licencias educativas, etcétera. El tipo de jornada, si es completa o parcial, que permite observar otro ángulo de la precariedad laboral, referido a la cantidad de horas que un trabajador dispone en un ámbito laboral, siempre y cuando demande mayor cantidad de horas a trabajar. Y el análisis de los impactos generacionales en la estructura ocupacional.

En los países seleccionados para el caso europeo, se tomó como referencia la tipología de los regímenes de bienestar propuesta por Gosta Esping-Andersen (1990). Hemos elegido cuatro países que representan cuatro tipos de estados de bienestar en Europa. España representa el modelo mediterráneo o latino, Suecia se situaría en el régimen socialdemócrata o escandinavo, Francia se ubica en el modelo de tipo corporativista, y Reino Unido en el modelo liberal.

Para Latinoamérica, inspirados en la clasificación de CEPAL (CEPAL 2010; Chávez Molina, 2013), también hemos elegido cuatro países, seleccionados a partir de la inversión social como porcentaje del PBI de cada país. Argentina y Brasil, por su orden de importancia para América del Sur, Ecuador, representando a los países andinos, y El Salvador por Centroamérica.

El análisis se inscribe en un contexto de instancias mundiales del aceleramiento de procesos de concentración del capital, y por ello, de pujas distributivas, que conllevan transformaciones sociales que operan en una variedad de formas y esferas que afectan las oportunidades sociales y laborales, las condiciones de vida, las biografías, la distribución de la riqueza y el poder y las perspectivas de movilidad social de los ciudadanos de ambos continentes.

En contraste con Europa, que vive un contexto de crisis e incertidumbre económica y que, junto a contratendencias político-ideológicas, ha resultado estar en un proceso de retroceso y desmantelamientos del estado de bienestar, que había constituido un eje central de la vida societal europea de protección social con posterioridad a la Segunda Guerra Mundial, América Latina, y en particular algunos países de la región, vive un proceso contrario. Ahí se da una 
mayor inclusión y protección de la vida societal de sus habitantes, partiendo de la base que dichos mecanismos de protección no existían, salvo para los asalariados formales, lo que se transforma en una verdadera paradoja, ya que, ante la restauración de los gobiernos neoliberales en la presente década, han debido incluir un piso de soporte societal novedoso para la región, que atenúa los planes de austeridad y ajuste, tan en boga hace tan solo pocas décadas atrás, y donde los soportes institucionales de protección no existían. Se generó una batería de políticas públicas basadas en la asignación de derechos ciudadanos a distintos actores sociales que anteriormente no eran reconocidos por el estado, tales como desempleados crónicos, pensionados sin contribuciones, amas de casa, enfermos crónicos que sufren discriminación por sus enfermedades (sida, diabetes, obesidad, etcétera), asignaciones a niños y niñas cuyos progenitores no tienen ingresos estables y formales, entre otros. A la luz de una mayor presencia estatal y de políticas públicas como mecanismos de protección social de grupos más marginales y desaventajados, también se fue configurando un proceso de desmercantilización de algunos aspectos de la vida social hasta hace poco dominados por mecanismos de mercado, tales como la salud, la vivienda social, la educación pública, no sin ello implicar pujas y confrontaciones políticas ideológicas entre los actores recurrentes que asumen los gobiernos o los confrontan en la oposición.

\section{La heterogeneidad laboral en el mercado europeo}

\subsection{La heterogeneidad estructural en América Latina. ¿Es posible observar a partir de ella Europa? Definición, la caracterización para América Latina y la reiteración sobre la pregunta si es utilizable para Europa.}

En un trabajo anterior (Chávez Molina, 2013), se sistematizaron los debates contemporáneos sobre la desigualdad basada en el enfoque centrado en las características heterogéneas de la producción, pero en el carácter estructural de dicha heterogeneidad. Generalmente, el término estructura se refiere a las características de las colectividades, los grupos y las sociedades, rasgos no imputables a los individuos y que ejercen un efecto constrictivo sobre las creencias y acciones de estos. La estructura tiene la característica de entenderse como el conjunto relativamente estable de las interrelaciones entre las diversas partes de una sociedad, más la distribución de estas partes según un orden dinámico (Feito Alonso, 1995).

La heterogeneidad estructural es un concepto que autores estructuralistas como Prebisch, Furtado y Pinto (Cimoli, 2005) utilizaron para destacar la concentración del progreso técnico y de sus frutos en América Latina. Con el mismo, aludían a la coexistencia de sectores, ramas o actividades donde la productividad del trabajo era elevada, es decir, similar a la que alcanzaban las economías de los países centrales, junto con otras ramas o actividades en que la productividad era mucho menor respecto a las registradas en las economías centrales (Pinto, 1969; Chena, 2009). 
Esta situación denota marcadas asimetrías entre segmentos de empresas y trabajadores, que se combinan con la concentración del empleo en estratos de muy baja productividad relativa (CEPAL, 2010). Las sociedades latinoamericanas presentan una profunda desigualdad, que se refleja en altos grados de concentración de la propiedad y una marcada heterogeneidad productiva. Y también con la existencia simultánea de sectores de productividad laboral media y alta, y un conjunto de segmentos en que la productividad del trabajo es muy baja. Por lo cual las brechas sociales no pueden explicarse sin entender la desigualdad en la calidad y productividad de los puestos de trabajo en y entre sectores de la actividad económica, la que se proyecta en rendimientos muy desiguales entre los trabajadores, el capital y el trabajo.

El criterio central que da cuenta de este funcionamiento es el tipo dominante de organización productiva, o sea, el sector monopólico-hegemónico. Sin embargo, que este sea el proceso de acumulación dominante en la actual fase de desarrollo capitalista no quiere decir que sea el único. Junto con él, coexisten un contingente amplio de pequeñas y medianas empresas que operan de manera mucho más parecida al estadio competitivo del capitalismo (Nun, 2001).

Esta base social productiva no solo configura a los individuos en el espacio social productivo, sino que además orienta las opciones de movilidad social, tanto a lo largo de su vida como en las comparaciones intergeneracionales. Esa es la intención para observar cuánto inciden estos factores explicativos para visualizar las probabilidades adecuadas de ascenso o descenso social. Pero, atención, ya no caracterizando el curso posible del individuo como un hecho aislado, sino al individuo en un hogar, espacio social de la contención, de la herencia, y de ambientación de valores cercanos en la vida social. Durante el periodo de estabilidad y crecimiento económico surgido en los países occidentales tras la Segunda Guerra Mundial, la sociología registró una imagen de las relaciones industriales que perdura, en buena medida, hasta nuestros días. Como es sabido, los trabajadores europeos disfrutaron no solo de grandes dosis de estabilidad en el empleo, sino de la posibilidad de promoción laboral y de una jubilación asegurada (Boltanski y Chiapello, 2002). No obstante, estos mercados laborales fueron sometidos a importantes crisis en los años setenta. La alta correlación entre inflación y desempleo, que predecía la curva de Phillips, comenzó a ser cuestionada dada la conjunción de altas tasas de paro e incremento de los precios. El éxito que hasta la fecha habían tenido las políticas inflacionarias en la lucha contra el desempleo demostró ser un éxito temporal (Friedman, 1975). Un nuevo fenómeno conocido como estanflación (Samuelson, 1974) comenzó a golpear la estabilidad de que hasta la fecha habían disfrutado las estructuras laborales de los países industrializados.

Ante semejante tesitura, los gobiernos implementaron políticas de desregulación laboral (Scharpf, 2002; Castel, 2001). Estas tenían como finalidad introducir elementos de flexibilidad que facilitaran la contratación de potenciales empleados (Adam y Canziani, 1998). Se configuró así un mercado laboral dual (Piore y Sabel, 1984) ya que mientras los trabajadores bien instalados en el mercado laboral se organizaban atendiendo a las rigidices propias de antes 
de la reforma, los nuevos trabajadores eran administrados según las nuevas medidas de flexibilidad entrantes. La lógica que estaba detrás de estas reformas era la de dotar de experiencia y habilidades a los nuevos trabajadores en un primer contrato que sirviera para estabilizar su trayectoria laboral futura. Sin embargo, se fue configurando un nuevo perfil laboral cuyos efectos no fueron deseados por la reforma (King y Rueda, 2008), el de un trabajador vulnerable con una débil vinculación al mercado laboral (Gallie y Paugam, 2000), cuyos trabajos siempre han sido temporales (Booth et al., 2002). El trabajo de Piore (1986) es fundamental en este sentido, pues señala que las nuevas economías de mercado responden a la disparidad de la demanda y la oferta entre los sectores económicos.

Así, los nuevos mercados laborales se segmentaron y dieron lugar a agregados de estables y precarios (Polavieja, 2004). Esta dualización no solo afectó a los jóvenes frente a los personas de mayor edad, sino en mayor medida a las mujeres frente a los hombres y los inmigrantes frente a los nativos. Del mismo modo, impactó de forma muy distinta a los miembros de distintas categorías profesionales y, asimismo, y esto es lo más importante, dentro de cada agregado profesional comenzaron a surgir diferencias horizontales incluso dentro de las ocupaciones de mayor prestigio y deseabilidad. Es, entonces, cuando los sociólogos empiezan a contemplar la posibilidad de pertenecer a una intraclase (Martucelli y Santiago, 2017) dentro de una determinada clase social.

Guy Standing (2011) va más allá aún que cualquiera de los autores que han estudiado el fenómeno y señala que estas categorías ocupacionales atípicas forman una clase social particular llamada el precariado. Como rasgo sobresaliente, el sociólogo británico destaca la adaptación de las expectativas a un empleo inestable y, por ello también, a una biografía insegura.

Las explicaciones a este fenómeno se han concentrado tanto en el lado de la demanda como de la oferta (Polavieja, 2004). La flexibilidad como respuesta a la volatilidad de las peticiones del mercado, el escaso desarrollo tecnológico de algunos sectores, la composición de la mano de obra, el tamaño de las empresas y el exceso legislación laboral (excesos costes de despido) han sido algunas de las explicaciones que se han dado. Como en otras regiones, la diferencia de productividad de los trabajadores se ha revelado como una de las causas aducidas.

Hasta la fecha, la forma de abordar el impacto de la flexibilidad en los mercados ha variado según sea el planteamiento de cada escuela teórica. Sin duda, una de las maneras más exitosas ha consistido en fraccionar los grupos ocupacionales en dos grupos diferentes atendiendo al hecho de si sufren en mayor o menor medida la precariedad. Partiendo del esquema de clase de Oesch (2006), Schwander y Häusermann (2013) construyen una subdivisión teóricamente muy sofisticada distinguiendo el empleo atípico por tipo de estado de bienestar.

No obstante, de lo que aquí se trata, como anteriormente hemos señalado, es de comprobar si la heterogeneidad estructural detectada en América Latina es un fenómeno que se pueda observar en las sociedades europeas o, al menos, en qué medida puede ser detectado. Nuestra hipótesis parte de la idea de que aquellos países europeos que han disfrutado de una industrialización tardía 
(Tortella, 1994) y de un desarrollo menor del estado de bienestar presentan niveles mayores de heterogeneidad estructural. Dicho de otra forma, la heterogeneidad es un continuo que se produce en mayor o en menor medida en todas las sociedades capitalistas. En qué medida la diferencia entre pequeña y gran empresa constituye una línea de fragmentación entre las clases sociales es la cuestión que trataremos de responder en este trabajo.

Esta sección se organiza en tres partes, que pasamos a explicar. En la primera de ellas, describimos la estructura de clases sociales empleando el esquema Solís, Chávez y Cobos (2016). Tomamos como referencia la tipología de los regímenes de bienestar propuesta por Gosta Esping-Andersen (1990). Hemos elegido cuatro países que representan cuatro tipos de estados de bienestar en Europa. España representa el modelo mediterráneo o latino; Suecia se situaría en el régimen socialdemócrata o escandinavo; Francia se ubica en el modelo de tipo corporativista, y Reino Unido en el modelo liberal. En segundo lugar, con la intención de comprobar el grado de heterogeneidad, contrastamos las diferencias de clase en los siguientes aspectos: ingresos, pobreza, tipo de contrato y jornada laboral. En tercer lugar, realizamos un bosquejo con estas dos últimas variables ubicándolas en un análisis de correspondencias múltiples. En cuarto lugar, contrastamos la variable ingresos con la clasificación Solís, Chávez y Cobos, y con la EGP la clasificación usual empleada en los análisis de las sociedades europeas. Los datos empleados para el análisis de clases en Europa es la Encuesta de Condiciones de Vida del año 2014 y la European Working Conditions Survey (2010 y 2015).

\section{La heterogeneidad de las clases sociales en Europa}

La tabla 1 muestra la estructura de clases europea según la clasificación de clases sociales propuesta por SCC (Solís, Chávez y Cobos, 2016). Como se ha dicho con anterioridad, esta clasificación tiene en cuenta las características distintivas de las relaciones laborales de los «países en desarrollo», a saber, la heterogeneidad estructural de los países latinoamericanos (Solís et al., 2016). A la luz de este nuevo algoritmo, ¿qué conclusiones pueden sacarse? Empecemos, primero, comparando la estructura de clases entre los países de Europa, y luego pasaremos a dar cuenta de las diferencias con América Latina.

Como se observa en la tabla 1, España es el país que tiene un menor porcentaje de directivos y profesionales en su conjunto (Ia, Ib, Ic y II). Solo un $23,2 \%$ de la fuerza laboral es empleada como clase servicio. En el resto de los países europeos, la proporción de la clase de servicio es mayor, especialmente en Suecia.

No existen grandes discrepancias en las ocupaciones de la clase no manual rutinaria (IIIa) de corte administrativo para los países europeos. No obstante, en lo que respecta al conjunto de la clase no manual rutinaria empleada en pequeñas empresas (IIIb-), este porcentaje es superior en España respecto al resto de países europeos, ya que alcanza casi el 3\%. Algunos autores han advertido de la existencia de la aparición de un proletariado de servicios (Esping- 
Tabla 1. Adaptación del EGP al SCC para España, Francia, Reino Unido y Suecia

\begin{tabular}{|c|c|c|c|c|}
\hline & España & Francia & Reino Unido & Suecia \\
\hline $\mathrm{I}+\mathrm{II}$ & $23,20 \%$ & $39,20 \%$ & $43,60 \%$ & $46,90 \%$ \\
\hline la Patrones y grandes directivos & 1,7 & 6,9 & 6,3 & 5,3 \\
\hline $\begin{array}{l}\text { Ib Profesionales asalariados y pequeños } \\
\text { patrones }\end{array}$ & 9,4 & 11,6 & 18,1 & 23,8 \\
\hline Ic Profesionales por cuenta propia & 2,5 & 2,3 & 3,1 & 3,4 \\
\hline II Low controllers & 9,6 & 18,4 & 16,1 & 14,4 \\
\hline Illab & $25,30 \%$ & $25,20 \%$ & $26,90 \%$ & $26,80 \%$ \\
\hline Illa Trabajadores no manuales de rutina & 10,5 & 11,1 & 9,5 & 8,2 \\
\hline $\begin{array}{l}\text { IIlb+ Trabajadores en ventas de grandes } \\
\text { comercios }\end{array}$ & 12,2 & 12,3 & 16,1 & 18,2 \\
\hline $\begin{array}{l}\text { Illb- Trabajadores en ventas de pequeños } \\
\text { comercios }\end{array}$ & 2,6 & 1,8 & 1,3 & 0,4 \\
\hline IVab & $9,40 \%$ & $4,60 \%$ & $6,10 \%$ & $4,90 \%$ \\
\hline IVa Pequeños patrones & 2,5 & 1,1 & 1,2 & 2,4 \\
\hline $\begin{array}{l}\text { IVb+ Trabajadores independientes } \\
\text { cualificados }\end{array}$ & 6,3 & 3,2 & 4,2 & 2,2 \\
\hline $\begin{array}{l}\text { IVb- Trabajadores independientes } \\
\text { no cualificados (sin agrícolas) }\end{array}$ & 0,6 & 0,3 & 0,7 & 0,3 \\
\hline $\mathrm{V}+\mathrm{VI}$ & $19,10 \%$ & $12,10 \%$ & $12,30 \%$ & $14,50 \%$ \\
\hline $\begin{array}{l}\mathrm{V}+\mathrm{VI}+\text { Trabajadores manuales cualificados } \\
\text { en grandes establecimientos }\end{array}$ & 17,8 & 11,2 & 11,7 & 14 \\
\hline $\begin{array}{l}\text { V+VI- Trabajadores manuales cualificados } \\
\text { en pequeños establecimientos }\end{array}$ & 1,3 & 0,9 & 0,6 & 0,5 \\
\hline VIla & $17,70 \%$ & $13,30 \%$ & $10,10 \%$ & $4,10 \%$ \\
\hline $\begin{array}{l}\text { VIla+ Trabajadores manuales no cualificados } \\
\text { en grandes establecimientos }\end{array}$ & 15,6 & 12,2 & 9,5 & 4 \\
\hline $\begin{array}{l}\text { VIla- Trabajadores manuales no cualificados } \\
\text { en pequeños establecimientos }\end{array}$ & 2,14 & 1,1 & 0,6 & 0,1 \\
\hline VIIb & $1,70 \%$ & $2 \%$ & $0,30 \%$ & $1 \%$ \\
\hline \multirow[t]{2}{*}{ IVc Trabajadores asalariados agrícolas } & $3,30 \%$ & $2,40 \%$ & $0,70 \%$ & $1,30 \%$ \\
\hline & $99,70 \%$ & $98,80 \%$ & $100,00 \%$ & $99,50 \%$ \\
\hline
\end{tabular}

Fuente: EUSILC, 2014.

Andersen, 1999; Castel, 1995). Utilizando otros instrumentos de medición, estos autores han señalado el aumento de la proporción de trabajadores destinados a la venta, distribución y los servicios de hostelería con sueldos bajos, contratos temporales e incluso horarios intempestivos.

Cuando observamos los pequeños empresarios, de nuevo, España tiene un mayor número de pequeños burgueses, sobre todo, de tipo cualificado (IVb+). En cambio, apenas se aprecian diferencias en la pequeña burguesía no cualificada (IVb-); son pocos los trabajadores que se encuentran ubicados en esta clase en los países europeos. En lo que respecta a los trabajadores cualificados y no cualificados, España y Suecia tienen una mayor presencia de obreros cualificados en grandes empresas $(\mathrm{V}+\mathrm{VI}+)$. 
Tabla 2. Adaptación del EGP al SCC para Argentina, Brasil, El Salvador y Perú

\begin{tabular}{|c|c|c|c|c|}
\hline & Argentina & Brasil & El Salvador & Perú \\
\hline$|+| I$ & $25,8 \%$ & $19,4 \%$ & $16,0 \%$ & $13,4 \%$ \\
\hline la Patrones y grandes directivos & $3,5 \%$ & $4,5 \%$ & $2,3 \%$ & $2,3 \%$ \\
\hline $\begin{array}{l}\text { Ib Profesionales asalariados y pequeños } \\
\text { patrones }\end{array}$ & $5,5 \%$ & $4,1 \%$ & $2,5 \%$ & $2,8 \%$ \\
\hline Ic Profesionales por cuenta propia & $5,0 \%$ & $1,1 \%$ & $4,9 \%$ & $0,5 \%$ \\
\hline II Low controllers & $11,9 \%$ & $9,6 \%$ & $6,2 \%$ & $7,8 \%$ \\
\hline Illab & $18,2 \%$ & $12,8 \%$ & $7,8 \%$ & $7,0 \%$ \\
\hline Illa Trabajadores no manuales de rutina & $13,5 \%$ & $8,1 \%$ & $4,3 \%$ & $5,3 \%$ \\
\hline $\begin{array}{l}\text { IIlb+ Trabajadores en ventas de grandes } \\
\text { comercios }\end{array}$ & $1,5 \%$ & $2,5 \%$ & $1,6 \%$ & $0,5 \%$ \\
\hline $\begin{array}{l}\text { IIlb- Trabajadores en ventas de pequeños } \\
\text { comercios }\end{array}$ & $3,1 \%$ & $2,2 \%$ & $1,9 \%$ & $1,2 \%$ \\
\hline IVab & $16,1 \%$ & $29,1 \%$ & $29,9 \%$ & $55,5 \%$ \\
\hline IVa Pequeños patrones & $3,7 \%$ & $3,9 \%$ & $5,4 \%$ & $8,9 \%$ \\
\hline $\begin{array}{l}\text { IVb+ Trabajadores independientes } \\
\text { cualificados }\end{array}$ & $8,2 \%$ & $10,8 \%$ & $11,4 \%$ & $15,9 \%$ \\
\hline $\begin{array}{l}\text { IVb- Trabajadores independientes } \\
\text { no cualificados (sin agrícolas) }\end{array}$ & $3,8 \%$ & $6,8 \%$ & $5,5 \%$ & $4,8 \%$ \\
\hline IVc Trabajadores independientes agrícolas & $0,4 \%$ & $7,6 \%$ & $7,5 \%$ & $25,9 \%$ \\
\hline $\mathrm{V}+\mathrm{VI}$ & $18,4 \%$ & $15,4 \%$ & $15,4 \%$ & $7,4 \%$ \\
\hline $\begin{array}{l}\mathrm{V}+\mathrm{VI}+\text { Trabajadores manuales cualificados } \\
\text { en grandes establecimientos }\end{array}$ & $13,6 \%$ & $11,5 \%$ & $9,6 \%$ & $5,0 \%$ \\
\hline $\begin{array}{l}\text { V+Vl- Trabajadores manuales cualificados } \\
\text { en pequeños establecimientos }\end{array}$ & $4,8 \%$ & $3,9 \%$ & $5,8 \%$ & $2,4 \%$ \\
\hline VIlla & $20,7 \%$ & $18,4 \%$ & $19,4 \%$ & $11,0 \%$ \\
\hline $\begin{array}{l}\text { VIla+ Trabajadores manuales no cualificados } \\
\text { en grandes establecimientos }\end{array}$ & $10,3 \%$ & $9,4 \%$ & $8,5 \%$ & $6,4 \%$ \\
\hline $\begin{array}{l}\text { VIla- Trabajadores manuales no cualificados } \\
\text { en pequeños establecimientos }\end{array}$ & $10,4 \%$ & $9,0 \%$ & $10,8 \%$ & $4,7 \%$ \\
\hline Vllb & $0,9 \%$ & $4,9 \%$ & $11,5 \%$ & $5,7 \%$ \\
\hline IVc Trabajadores asalariados agrícolas & $0,9 \%$ & $4,9 \%$ & $11,5 \%$ & $5,7 \%$ \\
\hline
\end{tabular}

Fuente: Encuestas Nacionales de Hogares, armonizadas por Colegio de México, 2016.

España presenta de nuevo una mayor cantidad de trabajadores no cualificados, tanto en el sector de la gran empresa (junto a Francia) como de la pequeña.

La clase social con menor peso en la estructura ocupacional en los cuatro países europeos se sitúa en el sector primario, lo que refleja el cambio en una estructura ocupacional de un sector eminentemente agrícola a un sector industrial, y tras poco tiempo y en mayor medida, de servicio. Comparados todos los países en conjunto, es en España donde esta clase tiene un mayor porcentaje de representación, tanto de pequeños propietarios agrícolas como de agricultores asalariados.

En Latinoamérica, como podemos observar en la tabla 2, la mayor presencia de directivos y profesionales se encuentra en Argentina, con el 25,8\%, 
seguida de Brasil, con el 19,4\%. Hay una mayor presencia de trabajadores asalariados de rutina en Argentina que en el resto de los países, con el 18,2\%, seguida de Brasil, con el 12,8\%, y más atrás Perú y El Salvador, con el 7\% aproximadamente. En la clase IIIab, se destaca una mayor representación en Argentina de los trabajadores de comercio en pequeños establecimientos.

En la clase IVab, Perú presenta los valores de representación de clase más elevados, sobre todo por la mayor presencia de trabajadores agrícolas, y pequeños patrones $(25,9 \% \text { y } 8,9 \% \text {, respectivamente })^{1}$. En las clases V+VI de los trabajadores manuales, producto de poseer un sector secundario más poderoso en la región, Argentina tiene la mayor representación relativa, donde se destaca la mayor presencia de trabajadores manuales cualificados en grandes establecimientos, $18,4 \%$, con relación a Brasil y El Salvador, 15\% aproximadamente. Situación que se repite en los trabajadores manuales no cualificados, aunque la proporción de los mismos en pequeños establecimientos se destaca en Argentina, el 10,4\%, sobre el resto de los países, aunque en los países de menor desarrollo de actividades secundarias, como El Salvador y Perú, presentan una mayor representación de trabajadores agrícolas asalariados.

A la hora de sintetizar, pueden ser resaltadas dos ideas. En primer lugar, pese a la convergencia producida en las estructuras sociales europeas, aún existen grandes diferencias en la estructura de clases de los países seleccionados. Sin duda, la principal diferencia se halla en la clase de servicio. Es España el país donde la clase de servicio disfruta de un porcentaje menor. Sin duda, habría que buscar la causa en la escasa demanda de mano de obra cualificada por parte de una estructura económica en la que el turismo, la agricultura y la construcción tienen un papel muy destacado.

Como puede apreciarse, y era del todo esperable, la estructura de clase de los países europeos es muy distinta a aquella de los países de América Latina. No obstante, en España la clase no manual rutinaria (IIIb-) y manual no cualificada en pequeñas empresas (VIIa-) alcanza porcentajes superiores a los del resto de Europa y recorta su distancia respecto a los países latinoamericanos de mayor industrialización. Pese a que en España existe un 16\% de trabajadores en pequeñas empresas, esta proporción no puede verse más que reducida cuando ha de ser repartida entre las clases: Ic, IVb+, IVb-, IIIb-, V+VI- y VIIa-, IVc y VIIb. Y es que hay que considerar un dato: solo un $5 \%$ de trabajadores de Suecia trabajan en establecimientos de cinco miembros. Este porcentaje sube a más de un 10\% en el caso de Reino Unido y Francia.

En ese sentido, América Latina muestra una mayor presencia de trabajadores manuales no cualificados en pequeños establecimientos, pero también una leve proporción mayor de trabajadores manuales no cualificados. En las comparaciones continentales se pronuncian en mayor medida las heterogeneidades estructurales en América Latina, siempre tomando esta apreciación en

1. Cabe destacar la menor participación relativa de trabajadores agrícolas de Argentina debido a los instrumentos de recolección de datos usados por las encuestas nacionales, principalmente por ser de carácter urbano. 
su carácter comparativo con los países centrales, como es el caso europeo y sus distintas vertientes estatales.

\section{Segunda dimensión: la pobreza relativa}

En este apartado, calculamos las tasas de pobreza relativa por clase social. Hemos computado la pobreza por debajo del umbral del $60 \%$ de la mediana de la renta disponible. En las tablas 3 y 4, mostramos en qué medida las clases sociales caen dentro de este umbral.

En ambas tablas realizamos dos tipos de análisis, siempre en términos comparativos, una mira intrapaíses y otra intercontinental, para apreciar tanto las heterogeneidades intrapaíses como interpaíses, a fin de dilucidar los aspectos diferenciales entre las clases.

En la primera mirada aparecen los siguientes resultados:

La mayor proporción de pobreza relativa europea se encuentra en los trabajadores independientes no cualificados, y luego los manuales no cualificados en

Tabla 3. Pobreza relativa, en los países europeos seleccionados

\begin{tabular}{|c|c|c|c|c|}
\hline & España & Francia & Reino Unido & Suecia \\
\hline la Patrones y grandes directivos & $7 \%$ & $1 \%$ & $7 \%$ & $3 \%$ \\
\hline $\begin{array}{l}\text { Ib Profesionales asalariados y pequeños } \\
\text { patrones }\end{array}$ & $3 \%$ & $3 \%$ & $5 \%$ & $4 \%$ \\
\hline Ic Profesionales por cuenta propia & $15 \%$ & $10 \%$ & $16 \%$ & $11 \%$ \\
\hline II Low controllers & $10 \%$ & $5 \%$ & $11 \%$ & $6 \%$ \\
\hline Illa Trabajadores no manuales de rutina & $8 \%$ & $7 \%$ & $13 \%$ & $9 \%$ \\
\hline $\begin{array}{l}\text { IIIb+ Trabajadores en ventas de grandes } \\
\text { comercios }\end{array}$ & $21 \%$ & $12 \%$ & $19 \%$ & $16 \%$ \\
\hline $\begin{array}{l}\text { Illb- Trabajadores en ventas de pequeños } \\
\text { comercios }\end{array}$ & $20 \%$ & $14 \%$ & $19 \%$ & $18 \%$ \\
\hline IVa Pequeños patrones & $20 \%$ & $10 \%$ & $32 \%$ & $16 \%$ \\
\hline $\begin{array}{l}\text { IVb+ Trabajadores independientes } \\
\text { cualificados }\end{array}$ & $28 \%$ & $20 \%$ & $21 \%$ & $21 \%$ \\
\hline $\begin{array}{l}\text { IVb- Trabajadores independientes } \\
\text { no cualificados (sin agrícolas) }\end{array}$ & $21 \%{ }^{*}$ & $28 \%$ ** & $27 \%$ & 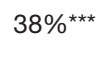 \\
\hline IVc Trabajadores independientes agrícolas & $21 \%$ & $11 \%$ & $18 \%$ & $10 \%$ \\
\hline $\begin{array}{l}\mathrm{V}+\mathrm{VI}+\text { Trabajadores manuales cualificados } \\
\text { en grandes establecimientos }\end{array}$ & $19 \% * *$ & $13 \%$ & $15 \%$ & $11 \%$ \\
\hline $\begin{array}{l}\text { V+VI- Trabajadores manuales cualificados } \\
\text { en pequeños establecimientos }\end{array}$ & $32 \%$ & $21 \%$ & $25 \%$ & $19 \%$ \\
\hline $\begin{array}{l}\text { Vlla+ Trabajadores manuales no cualificados } \\
\text { en grandes establecimientos }\end{array}$ & $25 \%$ & $23 \%$ & $23 \%$ & $12 \%$ \\
\hline $\begin{array}{l}\text { VIla- Trabajadores manuales no cualificados } \\
\text { en pequeños establecimientos }\end{array}$ & $35 \%$ & $16 \%$ & $17 \%$ & $25 \%$ \\
\hline IVc Trabajadores asalariados agrícolas & $14 \%$ & $21 \%$ & $20 \%$ & $40 \%$ \\
\hline Total & $18 \%$ & $10 \%$ & $14 \%$ & $10 \%$ \\
\hline
\end{tabular}

Fuente: EUSILC, 2014. 
Tabla 4. Pobreza relativa en los países latinoamericanos seleccionados

\begin{tabular}{|c|c|c|c|c|}
\hline & Argentina & Brasil & El Salvador & Ecuador \\
\hline la Patrones y grandes directivos & $2,7 \%$ & $1,9 \%$ & $2,3 \%$ & $3,8 \%$ \\
\hline $\begin{array}{l}\text { Ib Profesionales asalariados y pequeños } \\
\text { patrones }\end{array}$ & $3,2 \%$ & $2,5 \%$ & $2,3 \%$ & $0,0 \%$ \\
\hline Ic Profesionales por cuenta propia & $22,1 \%$ & $4,1 \%$ & $11,7 \%$ & $0,0 \%$ \\
\hline II Low controllers & $5,8 \%$ & $9,8 \%$ & $1,7 \%$ & $1,2 \%$ \\
\hline Illa Trabajadores no manuales de rutina & $5,5 \%$ & $15,0 \%$ & $2,3 \%$ & $0,0 \%$ \\
\hline $\begin{array}{l}\text { IIllb+ Trabajadores en ventas de grandes } \\
\text { comercios }\end{array}$ & $7,8 \%$ & $15,9 \%$ & $0,7 \%$ & $0,0 \%$ \\
\hline $\begin{array}{l}\text { Illb- Trabajadores en ventas de pequeños } \\
\text { comercios }\end{array}$ & $21,7 \%$ & $33,1 \%$ & $19,2 \%$ & $32,3 \%$ \\
\hline IVa Pequeños patrones & $11,5 \%$ & $3,1 \%$ & $26,6 \%$ & $8,2 \%$ \\
\hline $\begin{array}{l}\text { IVb+ Trabajadores independientes } \\
\text { cualificados }\end{array}$ & $30,4 \%$ & $20,1 \%$ & $23,2 \%$ & $9,0 \%$ \\
\hline $\begin{array}{l}\text { IVb- Trabajadores independientes } \\
\text { no cualificados (sin agrícolas) }\end{array}$ & $41,4 \%$ & $28,9 \%$ & $33,8 \%$ & $6,8 \%$ \\
\hline IVc Trabajadores independientes agrícolas & $53,2 \%$ & $55,6 \%$ & $91,3 \%$ & $42,5 \%$ \\
\hline $\begin{array}{l}\mathrm{V}+\mathrm{VI}+\text { Trabajadores manuales cualificados } \\
\text { en grandes establecimientos }\end{array}$ & $4,9 \%$ & $10,9 \%$ & $0,8 \%$ & $1,2 \%$ \\
\hline $\begin{array}{l}\text { V+VI- Trabajadores manuales cualificados } \\
\text { en pequeños establecimientos }\end{array}$ & $21,3 \%$ & $27,1 \%$ & $11,5 \%$ & $2,1 \%$ \\
\hline $\begin{array}{l}\text { Vlla+ Trabajadores manuales no cualificados } \\
\text { en grandes establecimientos }\end{array}$ & $17,1 \%$ & $24,5 \%$ & $3,2 \%$ & $2,5 \%$ \\
\hline $\begin{array}{l}\text { VIla- Trabajadores manuales no cualificados } \\
\text { en pequeños establecimientos }\end{array}$ & $46,1 \%$ & $44,2 \%$ & $23,5 \%$ & $9,8 \%$ \\
\hline IVc Trabajadores asalariados agrícolas & $31,0 \%$ & $53,1 \%$ & $23,7 \%$ & $24,4 \%$ \\
\hline Total & 24,0 & 28,9 & 35,0 & 28,0 \\
\hline
\end{tabular}

Fuente: Encuestas Nacionales de Hogares, armonizadas por Colegio de México, 2016.

grandes establecimientos, con porcentajes que duplican el promedio porcentual por país.

Y lo que esperábamos encontrar aparece con menor fuerza de lo esperado, con cierto comportamiento tendencial, donde los trabajadores de comercio, sin importar el tamaño del establecimiento, son más pobres que los no manuales de rutina, doblando los porcentajes.

El único segmento que presenta heterogeneidad por las condiciones del tamaño es la clase V+VI- Trabajadores manuales cualificados en pequeños establecimientos, donde los que están en establecimientos más pequeños tienen una condición de pobreza mayor. Y luego, en la clase VIIa-Trabajadores manuales no cualificados en pequeños establecimientos, solo se da esa diferencia en el caso de Suecia, pero no en el resto de los países.

Aquí, las divisiones intraclase deparan algunas discordancias más allá de las diferencias entre profesionales por cuenta propia o ajena que también hemos visto anteriormente con la renta. Salvo para Reino Unido, la pobreza impacta con mayor intensidad en los pequeños empresarios que tienen menor cuali- 
ficación, y para el caso español tanto los trabajadores cualificados como no cualificados presentan mayores porcentajes de pobreza cuando trabajan en pequeñas empresas.

¿Qué sucede en América Latina? La pobreza es mayúscula en ciertos segmentos de clases, por ejemplo los trabajadores agrícolas, que, en el caso de El Salvador, lo son casi todos, el 91\%.

$\mathrm{Al}$ observar las condiciones de la heterogeneidad, esto es patente en tres ámbitos específicos: los trabajadores en ventas de pequeños comercios, que triplican condiciones de pobreza relativa ante los trabajadores no manuales rutinarios y trabajadores no manuales en grandes comercios, y en tanto la mayor pobreza relativa de los IVb-Trabajadores independientes no cualificados (sin agricolas), junto a los IVc Trabajadores independientes agricolas, ante los trabajadores independientes cualificados.

Y luego V+VI+ Trabajadores manuales cualificados en grandes establecimientos y VIIa+ Trabajadores manuales no cualificados en grandes establecimientos. Sus condiciones de pobreza son mucho menores que en las clases ocupacionales clasificadas en V+VI- Trabajadores manuales cualificados en pequeños establecimientos y VIIa-Trabajadores manuales no cualificados en pequeños establecimientos.

El otro aspecto, intercontinental, señala claras diferencias en las estructuras de clases y sus condiciones de pobreza, por selección de países por continente. Por un lado, en el caso europeo son más tenues las condiciones de la heterogeneidad, aunque se manifiestan en algunos segmentos ocupacionales. La distribución de la pobreza tiende a ser más homogénea de acuerdo a las características de cada clase. En el caso latinoamericano, dicha situación es mucho más pronunciada; las características de la heterogeneidad son elocuentes, con diferencias entre países, como también se aprecia en Europa, pero con mayor pronunciación de pobreza relativa.

\section{Tercera dimensión: tipo de contrato}

Como hemos señalado más arriba, según Solís, Chávez y Cobos (2016), en la estructura productiva de América Latina existen dos sectores dispares. En el primero, la fuerza de trabajo disfruta de mejores condiciones laborales y salario fruto de su inserción en una economía formal-racional. En el segundo, las relaciones laborales no están definidas por las demandas productivas, sino más bien por factores de la oferta o, más sencillamente, por la lógica de supervivencia. La diferencia entre estos dos sectores se refleja en sus condiciones laborales. Por ello, es interesante abordar un análisis de la inseguridad laboral. Uno de los indicadores a los que literatura especializada ha prestado mayor atención es el tipo de contrato. En esta apartado, por razones obvias, no incluimos a aquellos que no venden su fuerza de trabajo, sino solo a los asalariados.

$\mathrm{Al}$ igual que en el anterior apartado, como se aprecia en la tabla 6, son los directivos los menos afectados por el contrato temporal, en bastante menor medida que los profesionales. Del mismo modo, existen importantes distancias 
Tabla 5. Tipo de contrato por clase social

\begin{tabular}{|c|c|c|c|c|}
\hline & España & Francia & Reino Unido & Suecia \\
\hline la & 6,25 & 3,38 & 1,49 & 3,92 \\
\hline $\mathrm{lb}$ & $20,48^{\star \star \star}$ & $11,48^{\star \star \star}$ & $4,66^{\star \star \star}$ & $11,11^{\star \star \star}$ \\
\hline ॥ & 24,12 & 9,7 & 3,09 & 7,71 \\
\hline Illa & 18,6 & 13,45 & 4,84 & 12,59 \\
\hline Illb+ & 39,42 & 19,91 & 3,43 & 22,45 \\
\hline Illb- & 37,34 & 15,95 & 4,07 & 17,86 \\
\hline $\mathrm{V}+\mathrm{VI}+$ & 29,02 & 15,58 & 2,59 & 12,54 \\
\hline V+VI- & 29,82 & 18,18 & 1,11 & 15,22 \\
\hline Vlla+ & 57,94 & 27,36 & 4,58 & 30,28 \\
\hline VIIa- & $44,64^{\star \star \star}$ & 22,89 & 2,33 & 50 \\
\hline VIlb & 41,84 & 31,39 & 6,25 & 46,81 \\
\hline
\end{tabular}

Fuente: EUSILC, 2014.

Tabla 6. Tipo de contrato por clase social

\begin{tabular}{lcccc}
\hline & Argentina & Brasil & Ecuador & El Salvador \\
\hline la & $7,0 \%$ & $17,0 \%$ & $10,7 \%$ & $8,5 \%$ \\
Ib & $5,3 \%$ & $10,7 \%$ & $48,7 \%$ & $41,4 \%$ \\
II & $8,7 \%$ & $12,2 \%$ & $16,5 \%$ & $16,3 \%$ \\
IIla & $10,6 \%$ & $11,9 \%$ & $27,7 \%$ & $18,4 \%$ \\
IIIb+ & $16,8 \%$ & $8,6 \%$ & $34,4 \%$ & $19,9 \%$ \\
IIIb- & $52,9 \%$ & $40,1 \%$ & $92,5 \%$ & $86,2 \%$ \\
V+VI+ & $13,5 \%$ & $7,6 \%$ & $22,1 \%$ & $18,4 \%$ \\
V+VI- & $58,1 \%$ & $52,3 \%$ & $86,4 \%$ & $89,4 \%$ \\
VIla+ & $26,8 \%$ & $10,4 \%$ & $41,9 \%$ & $31,5 \%$ \\
Vlla- & $74,9 \%$ & $62,8 \%$ & $90,6 \%$ & $93,9 \%$ \\
\hline
\end{tabular}

Fuente: Encuestas Nacionales de Hogares, armonizadas por Colegio de México, 2016.

entre el uso que se realiza del contrato temporal entre trabajadores no cualificados en grandes y pequeñas empresas en España. En líneas generales, habría que indicar que las cifras obtenidas para el caso español no tienen parangón con las europeas.

Para el caso latinoamericano, las contrataciones formales implican no solo protección estatal, sino en muchos casos el acompañamiento de la organización sindical, lo que permite una mayor disposición a presionar por mejores condiciones económicas en las pujas distributivas entre capital y trabajo.

La heterogeneidad de condiciones formalizadas en el tipo de contratación es notoria. En los asalariados de actividades no manuales de rutina, y empleados de comercio en establecimientos de más de 10 ocupados, triplica y hasta quintuplica porcentualmente sus condiciones de formalización con relación a los asalariados no manuales de comercio, en establecimientos de menos de 10 ocupados. 
Ocurre lo mismo con las clases $V+V I+$ Trabajadores manuales cualificados en grandes establecimientos, donde en Argentina el 13,5\% está sin contrato, el 7,6\% en Brasil, el 22,1\% en Ecuador, y el 18,4\% en El Salvador. Pero cuando observamos las clases V+VI- Trabajadores manuales cualificados en pequeños establecimientos, los niveles de ausencia de contratos llega a niveles del $58,1 \%$ en Argentina, el 52,3\% en Brasil, el 86,4\% en Ecuador y el 89,4\% en El Salvador.

Dicha situación se agudiza en los trabajadores manuales, como se observa en la tabla 7. VIIa + Trabajadores manuales no cualificados en grandes establecimientos, por un lado, muestra diferencias de formalización de los contratos entre Argentina y Brasil con relación a Ecuador y El Salvador. A esa primera diferencia, y como era esperable, se pronuncia una mayor distancia entre clases de cada país, con la clase VIIa-Trabajadores manuales no cualificados en pequeños establecimientos, y también se mantienen las distancias entre los países representativos del cono sur, y Ecuador junto a El Salvador.

\section{Contraste entre ambos esquemas para los países europeos y latinoamericanos}

Dado que las diferencias de renta constituyen la dimensión más importante de la desigualdad, regresamos esta variable según el esquema EGP y SCC. Se presentan, primero, los coeficientes de determinación de ambas clasificaciones y luego el contraste de los betas obtenidos empleando la prueba F de Fisher-Snedecor.

Los resultados son muy claros. El coeficiente de determinación del SCC mejora en tres puntos al esquema EGP en todos los países. Del mismo modo, el estadístico $F$ resulta ser significativo, pues ofrece una mejora sustancial del modelo en todos los países, especialmente para los países centroamericanos.

Un buen test añadido para comprobar el alcance de la clasificación SCC es confrontarla con la clasificación EGP. Como es bien sabido, es este el esquema de clases con un desarrollo mayor, al menos en el marco de las sociedades europeas. Nos centramos en la renta disponible familiar, puesto que es una de las variables de mayor importancia en sociedades asalariadas como las nuestras y una proxy de los recursos económicos realmente existentes.

En la tabla 6, se reflejan los resultados obtenidos tras una regresión simple de los ingresos con ambos esquemas. Hemos recogido los estadísticos $\mathrm{R}^{2}$, en

Tabla 7. Ajustes del esquema EGP y SCC, sendas regresiones con la renta disponible familiar para Europa

\begin{tabular}{lcccc}
\hline & EGP & SCC & & \\
& $\mathbf{R}^{2}$ & $\mathbf{R}^{2}$ & $\mathbf{F}$ & $\mathbf{P}$ \\
\hline España & 0,15 & 0,16 & 21,6 & 0,00 \\
Francia & 0,17 & 0,18 & 43,3 & 0,00 \\
Reino Unido & 0,12 & 0,12 & 24,0 & 0,00 \\
Suecia & 0,9 & 0,9 & 15,4 & 0,00 \\
\hline
\end{tabular}

Fuente: EUSILC, 2014. 
Tabla 8. Ajustes del esquema EGP y SCC, sendas regresiones con la renta disponible familiar para América Latina

\begin{tabular}{lccrc}
\hline & EGP & SCC & & \\
& $\mathbf{R}^{2}$ & $\mathbf{R}^{2}$ & $\mathbf{F}$ & $\mathbf{P}$ \\
\hline Argentina & 0,12 & 0,15 & 65,5 & 0,00 \\
Brasil & 0,20 & 0,22 & 180,7 & 0,00 \\
Ecuador & 0,24 & 0,27 & 121,1 & 0,00 \\
El Salvador & 0,21 & 0,24 & 65,2 & 0,00 \\
\hline
\end{tabular}

Fuente: Encuestas Nacionales de Hogares, armonizadas por Colegio de México, 2016.

primer lugar, y el contraste $\mathrm{F}$ en el segundo. En lo relativo al primero, los resultados son inequívocos. De acuerdo con el coeficiente de determinación, la clasificación SCC mejora muy poco en todos los países a pesar de los mayores grados de libertad empleados. Es algo mejor en España y Francia que en Reino Unido y en Suecia. El contraste F, en cambio, resulta ser significativo, aunque la mejora, quizás salvo en Francia, no resulta ser sustantiva en un grado elevado, sobre todo si se compara con los resultados de los países de América Latina.

\section{Conclusiones}

Nuestra idea giró en torno a observar dos fenómenos, por un lado las características de la estructura de clases en los países representativos de Europa y América Latina, y mostrar cómo es posible observar bajo un análisis diacrónico sus condiciones de heterogeneidad estructural si es que se manifiestan.

El afán de comparar presenta advertencias notorias, que tienen que ver con las conformaciones históricas, políticas y económicas muy disímiles que cada uno de los países, en sus entornos regionales, configuraron de manera distinta. Sin embargo, es posible observar ciertos rasgos comunes basados en la propia estructura de clases, en base a las clasificaciones ocupacionales, y así es posible generar una comparación entre variables similares, pero condicionadas por los aspectos generales mencionados anteriormente.

Este trabajo nos ha servido para responder a las preguntas planteadas al comienzo del mismo. En primer lugar, hemos demostrado la pertenencia de las subdivisiones de clase realizadas para América Latina.

Tanto la segmentación por cualificación de la pequeña burguesía como aquella fundamentada en la separación entre pequeña y mediana (y gran empresa) que atañe a los empleados en atención al público y a los trabajadores manuales generan resultados disímiles en materia de ingresos, pobreza, contrato. Hemos contrastado el esquema EGP con el SCC, comparando el impacto de sus coeficientes después de haber realizado una regresión con los ingresos. Los resultados muestran una sensible mejora sobre el esquema EGP.

El esquema propuesto por Solís, Chávez y Cobos cumple una función necesaria que es la obligada adaptación de los esquemas de clase a sus realidades institucionales. Sin la necesaria incrustación (Polanyi, 2003; Granovetter, 
2003), estos esquemas asumen la existencia de unos mercados no regulados (Polavieja, 2003).

Esta clasificación presenta, sin embargo, fuera del radio en la que fue creada, un alcance variable. El caso de España, da cuenta de disparidades en lo relativo a la pobreza y contrato. Esto es especialmente así para el caso de los trabajadores no cualificados en pequeñas empresas. Para Francia, los resultados son menos convincentes, y menos aún para Reino Unido y Suecia. Los contrastes F de Fisher-Snedecor, pese a que resultaron ser significativos, tuvieron un alcance, en comparación con América Latina, muy limitado, sobre todo para Reino Unido y Suecia.

Estas conclusiones deberían servirnos para dotar el esquema EGP de regulaciones sociohistóricas, ya que, de lo contrario, se hallaría «imbricado en un mundo institucionalmente desnudo" (Esping-Andersen, 1993: 8). La existencia de desigualdades estructurales, cuyo origen reside, en buena medida, en la acción colectiva, impide que estos esquemas «viajen» a través de distintos contextos constitucionales (Polavieja, 2004).

\section{Referencias bibliográficas}

ADAM, P. y CANZIANI, P. (1998). «Partial de-regulation: Fixed-term contracts in Italy and Spain». Centre for Economic Performance, London School of Economics and Political Science.

Boltanski, L. y ChiAPELlo, E. (2002). El nuevo espiritu del capitalismo (13). Ediciones Akal.

Booth, A. L.; Francesconi, M. y FranK, J. (2002). «Temporary jobs: stepping stones or dead ends?». The Economic Journal, 112 (480). <https://doi.org/10.1111/1468-0297.00043>.

CASTEL, R. (1995). «De la exclusión como estado a la vulnerabilidad como proceso". Archipiélago, 21, 27-36.

- (2001). «Empleo, exclusión y las nuevas cuestiones sociales». Desigualdad y globalización: cinco conferencias, 15-24. Facultad de Ciencias Sociales.

CEPAL, N. (2010). «La hora de la igualdad: brechas por cerrar, caminos por abrir». Trigésimo tercer período de sesiones de la CEPAL.

ChÁvez Molina, E. (2013). "Desigualdad y movilidad social en un contexto de heterogeneidad estructural: notas preliminares». En: CHÁVEZ MOLINA, Eduardo (compilador) y PLA, Jésica (colaboradora). Desigualdad y movilidad social en un contexto de heterogeneidad estructural: notas preliminares. CHÁVEZ MOLINA, Eduardo (compilador) y PLA, Jésica (colaboradora). Desigualdad y movilidad social en el mundo contemporáneo. Aportes empíricos y conceptuales. Argentina, China, España y Francia. Buenos Aires: Imago Mundi.

Chena, P. (2009). «Heterogeneidad estructural y distribución del ingreso. Una aproximación teórica a esta relación desde diferentes teorías económicas». En: 9o Congreso Nacional de Estudios del Trabajo: El trabajo como cuestión central. Asociación Argentina de Especialistas en Estudios del Trabajo (ASET). Buenos Aires: FCEUBA.

Cimoli, M. (2005). Heterogeneidad estructural, asimetrías tecnológicas y crecimiento en América Latina. CEPAL. 
ERIKSON, R.; GOLDTHORPE, J. H. y PORTOCARERO, L. (1979). «Intergenerational class mobility in three Western European societies: England, France and Sweden». The British Journal of Sociology, 30 (4), 415-441. <https://doi.org/10.1111/j.1468-4446.2009.01246.x>.

EsPIng-Andersen, G. (1990). The three worlds of welfare capitalism. Cambridge Polity Press. <https://doi.org/10.1007/978-3-531-90400-9_32>.

- (1999). Social foundations of postindustrial economies. Oxford University Press. <https://doi.org/10.1093/0198742002.001.0001>.

Feito Alonso, R. (1995). Estructura social contemporánea: las clases sociales en los países industrializados. Siglo Veintiuno de España.

FrIEDMAN, M. (1975). «Unemployment versus inflation?». Institute of Economic Affairs, Occasional Paper, 44.

Gallie, D. y Paugam, S. (2000). "The experience of unemployment in Europe: The debate». Age, 15 (24), 12-9. $<$ https://doi.org/10.1093/esr/17.4.469>.

GranovetTer, M. (2003). "La fuerza de los lazos débiles. Revisión de la teoría reticular. Análisis de redes sociales. Orígenes, teoría y práctica”. Colección monografia, 198, 196-230.

KInG, D. y RuedA, D. (2008). "Cheap labor: The new politics of 'bread and roses' in industrial democracies». Perspectives on Politics, 6 (2), 279-297. $<$ https://doi.org/10.1017/s1537592708080614>.

Martuccelli, D. y Santiago, J. (2017). El desafío sociológico hoy: individuo y retos sociales, 305. CIS-Centro de Investigaciones Sociológicas. $<$ https://doi.org/10.5477/cis/reis.149.131>.

Nun, J. (2001). Marginalidad y exclusión social. Fondo de Cultura Económica,.

OEsCH, D. (2006). Redrawing the class map. Stratification and institutions in Britain, Germany, Sweden and Switzerland. Basingstoke: Palgrave Macmillan. <https://10.1057/9780230504592>.

Pinto, A. (1969). Diagnóstico, estructura y esquemas de desarrollo en América Latina. Escuela Latinoamericana de Sociología-FLACSO.

Piore, M. J. (1986). «Perspectives on lobor market flexibility». Industrial Relations: A Journal of Economy and Society, 25 (2), 146-166.

Piore, M. y SABEL, C. (1984). The second industrial divide: Prospects for prosperity. Nueva York. <https://doi.org/10.1111/j.1468-232x.1986.tb00677.x>.

POLANYI, K. (2003). La gran transformación. Los orígenes políticos y económicos de nuestro tiempo. Fondo de Cultura Económica.

Polavieja, J. G. (2004). «El rompecabezas de la temporalidad: España en perspectiva comparada». VIII Congreso Español de Sociología. Alicante.

SAmuelson, P. A. (1974). «Worldwide stagflation». En: NAGATANi y Crowley (eds.). Collected Scientific Papers, 4. Cambridge: M.I.T. Press, 1977.

SCHARPF, F. W. (2002). "The European social model». JCMS: Journal of Common Market Studies, 40 (4), 645-670. <https://doi.org/10.1111/1468-5965.00392>.

SCHWANDER, H. y HäusermanN, S. (2013). "Who is in and who is out? A risk-based conceptualization of insiders and outsiders». Journal of European Social Policy, 23 (3), 248-269.

$<$ https://doi.org/10.1177/0958928713480064>. 
Solís, P.; ChÁvez Molina, E. y CoBos, D. (julio 2016). «Class structure, structural heterogeneity and living conditions in Latin America». Paper presented at the $3 \mathrm{rd}$ ISA Forum of Sociology.

StANDing, G. (2011). El precariado: una nueva clase social. Ediciones de Pasado y Presente. <https://doi.org/10.15446/rcs.v38n1.53288>.

Tortella Casares, G. (1994). El desarrollo de la España contemporánea. Historia económica de los siglos XIX y XX. Madrid: Alianza Editorial. 
